\title{
The Competitive Effects of Entry: Evidence from Supercenter Expansion
}

Online Appendix

\author{
Peter Arcidiacono, Paul B. Ellickson, Carl F. Mela, and John D. Singleton*
}

June 2019

Table A1: Trends in the Revenue Effect of Supercenters

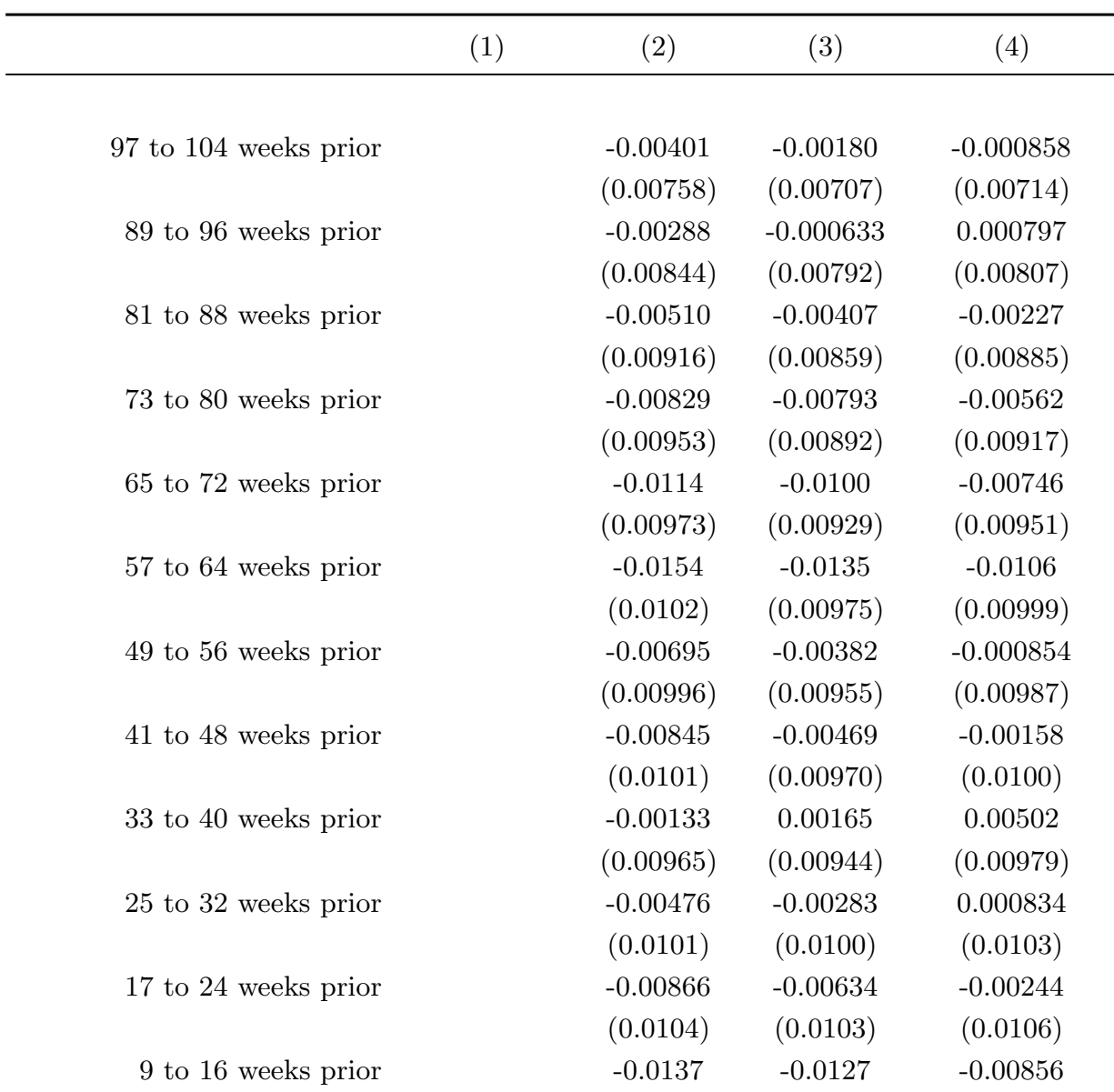

*Arcidiacono: Department of Economics, Duke University \& NBER (psarcidi@econ.duke.edu); Ellickson: Simon Business School, University of Rochester (paul.ellickson@simon.rochester.edu); Mela: Fuqua School of Business, Duke University (mela@duke.edu); Singleton: Department of Economics, University of Rochester (john.singleton@rochester.edu). 


\begin{tabular}{|c|c|c|c|c|}
\hline \multirow{3}{*}{1 to 8 weeks prior } & & $(0.0106)$ & $(0.0105)$ & $(0.0108)$ \\
\hline & & -0.00630 & -0.00818 & -0.00434 \\
\hline & & $(0.0105)$ & $(0.0105)$ & $(0.0108)$ \\
\hline \multirow[t]{2}{*}{ Week of entry } & $-0.0371^{* * *}$ & $-0.0388^{* * *}$ & $-0.0354^{* *}$ & $-0.0346^{* *}$ \\
\hline & $(0.0138)$ & $(0.0141)$ & $(0.0144)$ & $(0.0140)$ \\
\hline \multirow[t]{2}{*}{1 to 8 weeks post } & $-0.146^{* * *}$ & $-0.149^{* * *}$ & $-0.144^{* * *}$ & $-0.140 * * *$ \\
\hline & $(0.0291)$ & $(0.0296)$ & $(0.0304)$ & $(0.0301)$ \\
\hline \multirow[t]{2}{*}{9 to 16 weeks post } & $-0.152^{* * *}$ & $-0.156^{* * *}$ & $-0.152^{* * *}$ & $-0.147^{* * *}$ \\
\hline & $(0.0294)$ & $(0.0299)$ & $(0.0307)$ & $(0.0304)$ \\
\hline \multirow[t]{2}{*}{17 to 24 weeks post } & $-0.150 * * *$ & $-0.153^{* * *}$ & $-0.150^{* * *}$ & $-0.145^{* * *}$ \\
\hline & $(0.0292)$ & $(0.0297)$ & $(0.0305)$ & $(0.0302)$ \\
\hline \multirow[t]{2}{*}{25 to 32 weeks post } & $-0.150^{* * *}$ & $-0.154^{* * *}$ & $-0.150 * * *$ & $-0.145^{* * *}$ \\
\hline & $(0.0292)$ & $(0.0298)$ & $(0.0306)$ & $(0.0303)$ \\
\hline \multirow[t]{2}{*}{33 to 40 weeks post } & $-0.151^{* * *}$ & $-0.155^{* * *}$ & $-0.151^{* * *}$ & $-0.145^{* * *}$ \\
\hline & $(0.0292)$ & $(0.0297)$ & $(0.0306)$ & $(0.0303)$ \\
\hline \multirow[t]{2}{*}{41 to 48 weeks post } & $-0.152^{* * *}$ & $-0.156^{* * *}$ & $-0.152^{* * *}$ & $-0.146^{* * *}$ \\
\hline & $(0.0293)$ & $(0.0299)$ & $(0.0307)$ & $(0.0304)$ \\
\hline \multirow[t]{2}{*}{49 to 56 weeks post } & $-0.156^{* * *}$ & $-0.160 * * *$ & $-0.155^{* * *}$ & $-0.148 * * *$ \\
\hline & $(0.0294)$ & $(0.0300)$ & $(0.0309)$ & $(0.0306)$ \\
\hline \multirow[t]{2}{*}{57 to 64 weeks post } & $-0.152^{* * *}$ & $-0.156^{* * *}$ & $-0.152^{* * *}$ & $-0.145^{* * *}$ \\
\hline & $(0.0295)$ & $(0.0301)$ & $(0.0311)$ & $(0.0308)$ \\
\hline \multirow[t]{2}{*}{65 to 72 weeks post } & $-0.144^{* * *}$ & $-0.148^{* * *}$ & $-0.145^{* * *}$ & $-0.138^{* * *}$ \\
\hline & $(0.0297)$ & $(0.0303)$ & $(0.0313)$ & $(0.0310)$ \\
\hline \multirow[t]{2}{*}{73 to 80 weeks post } & $-0.147^{* * *}$ & $-0.151^{* * *}$ & $-0.147^{* * *}$ & $-0.139 * * *$ \\
\hline & $(0.0297)$ & $(0.0304)$ & $(0.0313)$ & $(0.0311)$ \\
\hline \multirow[t]{2}{*}{81 to 88 weeks post } & $-0.148^{* * *}$ & $-0.152^{* * *}$ & $-0.148^{* * *}$ & $-0.141^{* * *}$ \\
\hline & $(0.0296)$ & $(0.0303)$ & $(0.0311)$ & $(0.0309)$ \\
\hline \multirow[t]{2}{*}{89 to 96 weeks post } & $-0.149^{* * *}$ & $-0.153^{* * *}$ & $-0.149 * * *$ & $-0.141^{* * *}$ \\
\hline & $(0.0296)$ & $(0.0302)$ & $(0.0310)$ & $(0.0309)$ \\
\hline \multirow[t]{2}{*}{97 to 104 weeks post } & $-0.149 * * *$ & $-0.153^{* * *}$ & $-0.148^{* * *}$ & $-0.140 * * *$ \\
\hline & $(0.0296)$ & $(0.0302)$ & $(0.0310)$ & $(0.0309)$ \\
\hline \multirow[t]{2}{*}{105 weeks or more post } & $-0.143^{* * *}$ & $-0.147 * * *$ & $-0.146^{* * *}$ & $-0.135^{* * *}$ \\
\hline & $(0.0296)$ & $(0.0302)$ & $(0.0313)$ & $(0.0313)$ \\
\hline \multirow{2}{*}{1 Mile $\leq \# \mathrm{WM}<3$ Miles } & $0.0783^{* *}$ & $0.0780^{* *}$ & $0.0671^{*}$ & $0.0664^{*}$ \\
\hline & $(0.0386)$ & $(0.0386)$ & $(0.0386)$ & $(0.0385)$ \\
\hline \multirow[t]{2}{*}{3 Miles $\leq \# \mathrm{WM}<5$ Miles } & $0.0962^{* * *}$ & $0.0957 * * *$ & $0.0934^{* * *}$ & $0.0968^{* * *}$ \\
\hline & $(0.0326)$ & $(0.0327)$ & $(0.0329)$ & $(0.0318)$ \\
\hline \multirow[t]{2}{*}{5 Miles $\leq \# \mathrm{WM}<7$ Miles } & $0.126^{* * *}$ & $0.126^{* * *}$ & $0.117^{* * *}$ & $0.112^{* * *}$ \\
\hline & $(0.0316)$ & $(0.0317)$ & $(0.0329)$ & $(0.0332)$ \\
\hline Period FE & $\mathrm{Y}$ & $\mathrm{Y}$ & $\mathrm{Y}$ & $\mathrm{Y}$ \\
\hline Store FE & $\mathrm{Y}$ & $\mathrm{Y}$ & $\mathrm{Y}$ & $\mathrm{Y}$ \\
\hline Market Trends & $\mathrm{N}$ & $\mathrm{N}$ & $\mathrm{Y}$ & $\mathrm{Y}$ \\
\hline Treat Group Trends & $\mathrm{N}$ & $\mathrm{N}$ & $\mathrm{N}$ & $\mathrm{Y}$ \\
\hline
\end{tabular}


Notes: Table presents results of regressing incumbent supermarket log revenue on Walmart Supercenter exposure allowing for heterogeneity in time to or from entry. Less than 1 mile is excluded driving distance exposure category. Trends components to Supercenter effects apply to all entries within 7 miles. Specification also controls for number of Supercenters in 7 to 9 and 9 to 11 distance bands. 756,097 store-week observations. ${ }^{* * *} \mathrm{p}<0.01, * * \mathrm{p}<0.05$,

$* \mathrm{p}<0.1$. Standard errors clustered by store in parentheses. Treatment groups are defined by total Supercenter exposures. Markets are IRI designated markets. All trend controls are quadratic.

Table A2: Trends in the Price Effect of Supercenters

\begin{tabular}{|c|c|c|c|c|}
\hline & (1) & $(2)$ & $(3)$ & $(4)$ \\
\hline 97 to 104 weeks prior & & $\begin{array}{c}-0.00419^{* *} \\
(0.00192)\end{array}$ & $\begin{array}{l}-0.00217 \\
(0.00171)\end{array}$ & $\begin{array}{l}-0.00225 \\
(0.00170)\end{array}$ \\
\hline 89 to 96 weeks prior & & $\begin{array}{c}-0.00432^{* *} \\
(0.00203)\end{array}$ & $\begin{array}{l}-0.00264 \\
(0.00180)\end{array}$ & $\begin{array}{l}-0.00275 \\
(0.00179)\end{array}$ \\
\hline 81 to 88 weeks prior & & $\begin{array}{c}-0.00488^{* *} \\
(0.00206)\end{array}$ & $\begin{array}{c}-0.00419 * * \\
(0.00184)\end{array}$ & $\begin{array}{c}-0.00428^{* *} \\
(0.00183)\end{array}$ \\
\hline 73 to 80 weeks prior & & $\begin{array}{c}-0.00437^{* *} \\
(0.00215)\end{array}$ & $\begin{array}{c}-0.00389 * * \\
(0.00187)\end{array}$ & $\begin{array}{c}-0.00400^{* *} \\
(0.00186)\end{array}$ \\
\hline 65 to 72 weeks prior & & $\begin{array}{c}-0.00456^{* *} \\
(0.00219)\end{array}$ & $\begin{array}{c}-0.00400^{* *} \\
(0.00194)\end{array}$ & $\begin{array}{r}-0.00415^{* *} \\
(0.00195)\end{array}$ \\
\hline 57 to 64 weeks prior & & $\begin{array}{c}-0.00367^{*} \\
(0.00222)\end{array}$ & $\begin{array}{l}-0.00297 \\
(0.00196)\end{array}$ & $\begin{array}{l}-0.00317 \\
(0.00196)\end{array}$ \\
\hline 49 to 56 weeks prior & & $\begin{array}{l}-0.00327 \\
(0.00230)\end{array}$ & $\begin{array}{l}-0.00247 \\
(0.00200)\end{array}$ & $\begin{array}{l}-0.00267 \\
(0.00201)\end{array}$ \\
\hline 41 to 48 weeks prior & & $\begin{array}{l}-0.00253 \\
(0.00239)\end{array}$ & $\begin{array}{l}-0.00176 \\
(0.00202)\end{array}$ & $\begin{array}{l}-0.00195 \\
(0.00204)\end{array}$ \\
\hline 33 to 40 weeks prior & & $\begin{array}{l}-0.00324 \\
(0.00245)\end{array}$ & $\begin{array}{l}-0.00230 \\
(0.00208)\end{array}$ & $\begin{array}{l}-0.00245 \\
(0.00213)\end{array}$ \\
\hline 25 to 32 weeks prior & & $\begin{array}{l}-0.00109 \\
(0.00250)\end{array}$ & $\begin{array}{l}-0.000227 \\
(0.00213)\end{array}$ & $\begin{array}{l}-0.000381 \\
(0.00218)\end{array}$ \\
\hline 17 to 24 weeks prior & & $\begin{array}{l}0.000875 \\
(0.00257)\end{array}$ & $\begin{array}{c}0.00212 \\
(0.00217)\end{array}$ & $\begin{array}{c}0.00197 \\
(0.00223)\end{array}$ \\
\hline 9 to 16 weeks prior & & $\begin{array}{l}-0.00153 \\
(0.00262)\end{array}$ & $\begin{array}{l}0.000325 \\
(0.00213)\end{array}$ & $\begin{array}{l}0.000177 \\
(0.00219)\end{array}$ \\
\hline 1 to 8 weeks prior & & $\begin{array}{c}-0.00452^{*} \\
(0.00272)\end{array}$ & $\begin{array}{l}-0.00245 \\
(0.00220)\end{array}$ & $\begin{array}{l}-0.00261 \\
(0.00227)\end{array}$ \\
\hline Week of entry & $\begin{array}{c}0.00244^{*} \\
(0.00140)\end{array}$ & $\begin{array}{c}0.00171 \\
(0.00167)\end{array}$ & $\begin{array}{c}0.00198 \\
(0.00143)\end{array}$ & $\begin{array}{c}0.00173 \\
(0.00144)\end{array}$ \\
\hline 1 to 8 weeks post & $\begin{array}{c}-0.00365^{*} \\
(0.00198)\end{array}$ & $\begin{array}{c}-0.00526^{*} \\
(0.00285)\end{array}$ & $\begin{array}{l}-0.00303 \\
(0.00229)\end{array}$ & $\begin{array}{l}-0.00325 \\
(0.00236)\end{array}$ \\
\hline 9 to 16 weeks post & $\begin{array}{l}-0.00192 \\
(0.00207)\end{array}$ & $\begin{array}{l}-0.00355 \\
(0.00293)\end{array}$ & $\begin{array}{c}-0.000828 \\
(0.00234)\end{array}$ & $\begin{array}{l}-0.00102 \\
(0.00241)\end{array}$ \\
\hline 17 to 24 weeks post & $\begin{array}{l}-0.00193 \\
(0.00215)\end{array}$ & $\begin{array}{l}-0.00362 \\
(0.00299)\end{array}$ & $\begin{array}{r}-0.000746 \\
(0.00241)\end{array}$ & $\begin{array}{r}-0.000957 \\
(0.00250)\end{array}$ \\
\hline
\end{tabular}




\begin{tabular}{|c|c|c|c|c|}
\hline 25 to 32 weeks post & $\begin{array}{c}-0.000343 \\
(0.00216)\end{array}$ & $\begin{array}{l}-0.00203 \\
(0.00299)\end{array}$ & $\begin{array}{c}0.000854 \\
(0.00247)\end{array}$ & $\begin{array}{c}0.000626 \\
(0.00258)\end{array}$ \\
\hline \multirow[t]{2}{*}{33 to 40 weeks post } & -0.00130 & -0.00297 & 0.000722 & 0.000478 \\
\hline & $(0.00218)$ & $(0.00300)$ & $(0.00245)$ & $(0.00255)$ \\
\hline \multirow[t]{2}{*}{41 to 48 weeks post } & -0.00362 & $-0.00527^{*}$ & -0.00141 & -0.00170 \\
\hline & $(0.00222)$ & $(0.00303)$ & $(0.00249)$ & $(0.00260)$ \\
\hline \multirow[t]{2}{*}{49 to 56 weeks post } & $-0.00422^{*}$ & $-0.00592^{*}$ & -0.00170 & -0.00193 \\
\hline & $(0.00235)$ & $(0.00314)$ & $(0.00266)$ & $(0.00277)$ \\
\hline \multirow[t]{2}{*}{57 to 64 weeks post } & -0.00366 & $-0.00536^{*}$ & -0.000683 & -0.000936 \\
\hline & $(0.00237)$ & $(0.00314)$ & $(0.00260)$ & $(0.00273)$ \\
\hline \multirow[t]{2}{*}{65 to 72 weeks post } & $-0.00408^{*}$ & $-0.00576^{*}$ & -0.000770 & -0.00103 \\
\hline & $(0.00242)$ & $(0.00321)$ & $(0.00262)$ & $(0.00276)$ \\
\hline \multirow[t]{2}{*}{73 to 80 weeks post } & $-0.00539^{* *}$ & $-0.00708^{* *}$ & -0.00163 & -0.00181 \\
\hline & $(0.00253)$ & $(0.00330)$ & $(0.00268)$ & $(0.00284)$ \\
\hline \multirow[t]{2}{*}{81 to 88 weeks post } & $-0.00472^{*}$ & $-0.00646^{* *}$ & -0.000794 & -0.000947 \\
\hline & $(0.00254)$ & $(0.00329)$ & $(0.00272)$ & $(0.00289)$ \\
\hline \multirow[t]{2}{*}{89 to 96 weeks post } & $-0.00622^{* *}$ & $-0.00793^{* *}$ & -0.00203 & -0.00217 \\
\hline & $(0.00264)$ & $(0.00338)$ & $(0.00277)$ & $(0.00294)$ \\
\hline \multirow[t]{2}{*}{97 to 104 weeks post } & $-0.00634^{* *}$ & $-0.00802^{* *}$ & -0.00169 & -0.00184 \\
\hline & $(0.00274)$ & $(0.00345)$ & $(0.00284)$ & $(0.00304)$ \\
\hline \multirow[t]{2}{*}{105 weeks or more post } & $-0.0121^{* * *}$ & $-0.0139 * * *$ & -0.000911 & -0.000705 \\
\hline & $(0.00294)$ & $(0.00353)$ & $(0.00296)$ & $(0.00323)$ \\
\hline Period FE & $\mathrm{Y}$ & $\mathrm{Y}$ & $\mathrm{Y}$ & $\mathrm{Y}$ \\
\hline Store FE & $\mathrm{Y}$ & $\mathrm{Y}$ & $\mathrm{Y}$ & $\mathrm{Y}$ \\
\hline Market Trends & $\mathrm{N}$ & $\mathrm{N}$ & $\mathrm{Y}$ & $\mathrm{Y}$ \\
\hline Treat Group Trends & $\mathrm{N}$ & $\mathrm{N}$ & $\mathrm{N}$ & $\mathrm{Y}$ \\
\hline
\end{tabular}

Notes: Table presents results of regressing incumbent supermarket log prices on Walmart Supercenter exposure allowing for heterogeneity in time to or from entry. Trend components to Supercenter effects apply to all entries within 7 miles. 11,253,196 storecategory-week observations. ${ }^{* * *} \mathrm{p}<0.01,{ }^{* *} \mathrm{p}<0.05,{ }^{*} \mathrm{p}<0.1$. Standard errors clustered by store in parentheses. Treatment groups are defined by total Supercenter exposures. Markets are IRI designated markets. All trends are quadratic. 


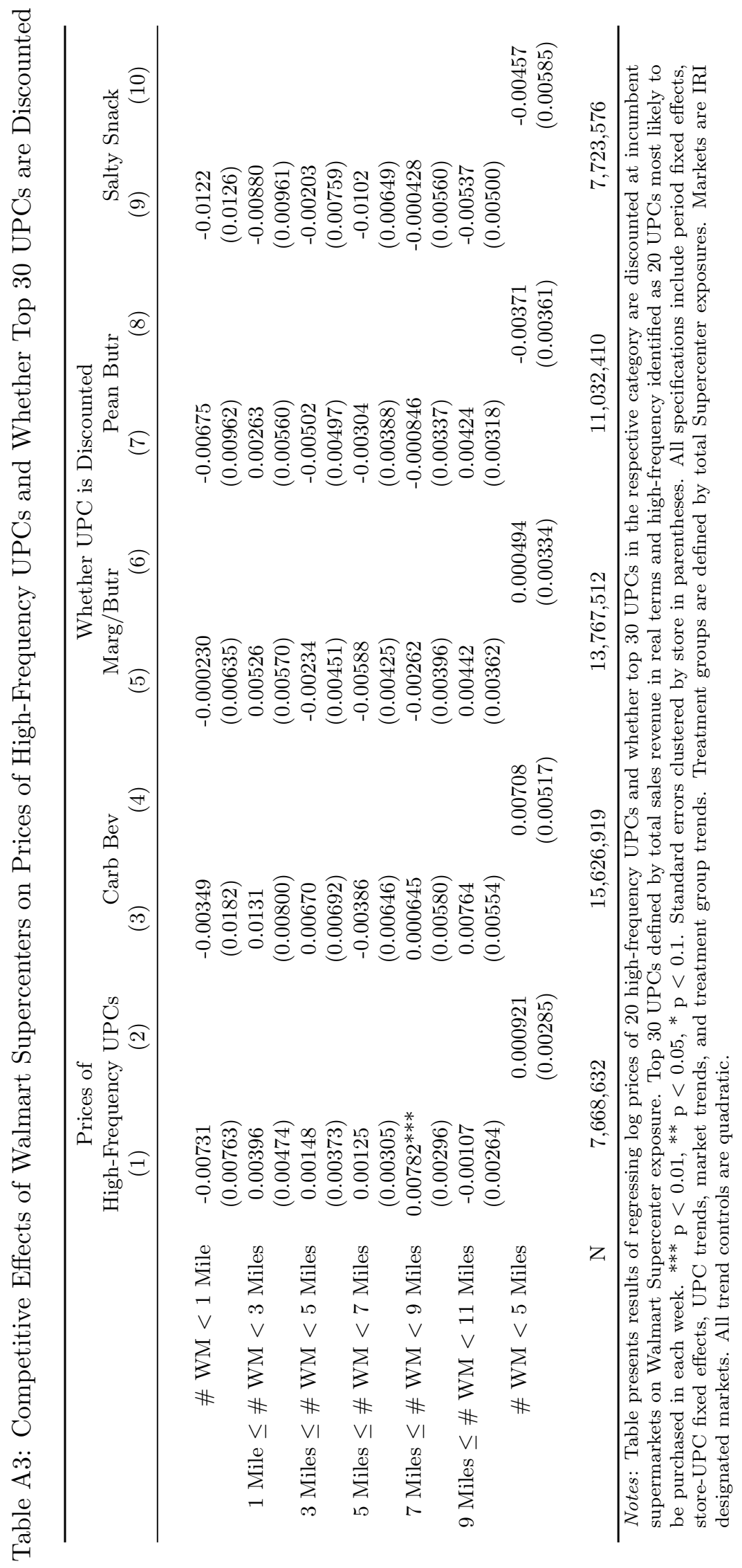


Table A4: Frozen Pizza UPC Price Summaries

\begin{tabular}{rcccccccc}
\hline \multicolumn{1}{c}{ Brand } & Share & Mean & SD & p10 & p25 & Median & p75 & p90 \\
\hline Amy's Kitchen & $1.05 \%$ & 6.89 & 1.58 & 5.23 & 5.89 & 6.65 & 7.64 & 8.81 \\
CA Pizza Kitchen & $4.07 \%$ & 6.12 & 1.09 & 4.75 & 5.49 & 6.13 & 6.77 & 7.40 \\
Stouffer's & $7.31 \%$ & 5.22 & 1.75 & 3.14 & 3.79 & 4.94 & 6.57 & 7.59 \\
Other & $13.08 \%$ & 4.23 & 2.06 & 1.90 & 2.51 & 3.93 & 5.64 & 6.96 \\
Freschetta & $7.65 \%$ & 3.90 & 1.29 & 2.51 & 2.95 & 3.64 & 4.68 & 5.67 \\
Celeste & $3.61 \%$ & 3.48 & 1.28 & 2.16 & 2.48 & 3.10 & 4.22 & 5.48 \\
DiGiorno & $17.53 \%$ & 3.48 & 1.01 & 2.35 & 2.74 & 3.29 & 4.09 & 4.88 \\
Red Baron & $12.83 \%$ & 3.45 & 1.09 & 2.11 & 2.65 & 3.33 & 4.12 & 4.85 \\
Tony's & $4.81 \%$ & 2.80 & 0.88 & 1.78 & 2.12 & 2.63 & 3.40 & 4.00 \\
Tombstone & $9.23 \%$ & 2.79 & 0.77 & 1.87 & 2.22 & 2.70 & 3.32 & 3.79 \\
Jack's & $5.36 \%$ & 2.47 & 0.50 & 1.84 & 2.13 & 2.44 & 2.79 & 3.11 \\
Private Label & $8.55 \%$ & 2.41 & 1.07 & 1.53 & 1.72 & 2.05 & 2.68 & 4.07 \\
Totino's & $4.92 \%$ & 1.90 & 0.58 & 1.33 & 1.50 & 1.79 & 2.16 & 2.58 \\
\hline
\end{tabular}

Notes: Table presents summary statistics for frozen pizza UPC prices (in January 2001 dollars) by brand. Share is overall revenue share of brand. Each observation represents a store-UPC-week.

Table A5: Spaghetti Sauce UPC Price Summaries

\begin{tabular}{rcccccccc}
\hline \multicolumn{1}{c}{ Brand } & Share & Mean & SD & p10 & p25 & Median & p75 & p90 \\
\hline Other & $10.50 \%$ & 3.20 & 2.63 & 1.19 & 1.73 & 2.48 & 3.60 & 5.43 \\
Emeril & $0.85 \%$ & 2.09 & 0.46 & 1.48 & 1.74 & 2.11 & 2.44 & 2.68 \\
Bertolli & $5.50 \%$ & 1.92 & 0.64 & 1.32 & 1.46 & 1.73 & 2.27 & 2.78 \\
Classico & $12.56 \%$ & 1.86 & 0.77 & 1.17 & 1.37 & 1.61 & 2.13 & 2.79 \\
Newman's Own & $3.33 \%$ & 1.56 & 0.34 & 1.19 & 1.35 & 1.52 & 1.71 & 1.98 \\
Barilla & $4.36 \%$ & 1.50 & 0.42 & 1.11 & 1.27 & 1.44 & 1.66 & 1.89 \\
Prego & $18.96 \%$ & 1.21 & 0.29 & 0.88 & 1.00 & 1.17 & 1.39 & 1.61 \\
Ragu & $25.78 \%$ & 1.17 & 0.39 & 0.80 & 0.90 & 1.08 & 1.36 & 1.72 \\
Private Label & $6.89 \%$ & 1.10 & 0.97 & 0.52 & 0.64 & 0.87 & 1.24 & 1.73 \\
Rinaldi & $4.31 \%$ & 1.06 & 0.50 & 0.59 & 0.76 & 0.94 & 1.16 & 1.71 \\
Del Monte & $1.42 \%$ & 0.63 & 0.13 & 0.48 & 0.55 & 0.60 & 0.72 & 0.82 \\
Hunt's & $5.54 \%$ & 0.62 & 0.14 & 0.49 & 0.53 & 0.60 & 0.70 & 0.80 \\
\hline
\end{tabular}

Notes: Table presents summary statistics for spaghetti sauce UPC prices (in January 2001 dollars) by brand. Share is overall revenue share of brand. Each observation represents a store-UPC-week. 
Table A6: Competitive Effects of Walmart Supercenters by Brand: Spaghetti Sauce

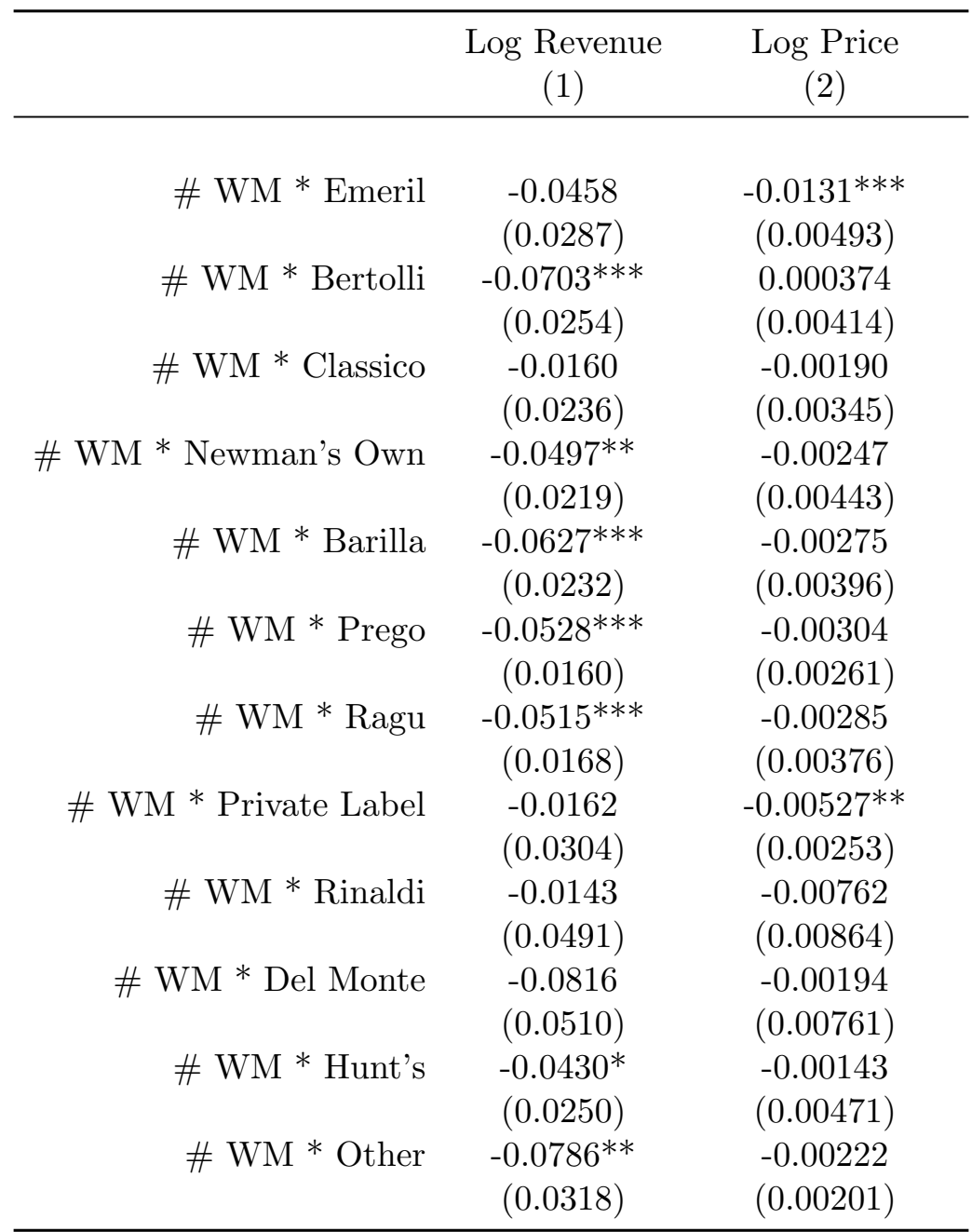

Notes: Table presents results of regressing incumbent supermarket log revenues and log prices for spaghetti sauce on Walmart Supercenter exposure allowing for heterogeneity by brand. $\mathrm{WM}=1$ for Supercenter exposures within 5 miles. 7,359,421 store-brand-week observations in column (1) and $75,116,201$ store-UPC-week observations in column $(2)^{* * *} \mathrm{p}<0.01$, ** $\mathrm{p}<$ $0.05, * \mathrm{p}<0.1$. Standard errors clustered by store in parentheses. Markets are IRI designated markets. Standard errors clustered by store in parentheses. Column (1) includes period fixed effects, store-category fixed effects, category trends, market trends, and treatment group trends. Treatment groups are defined by total Supercenter exposures. Markets are IRI designated markets. All trends are quadratic. Column (2) includes store-UPC fixed effects and market-UPC-period fixed effects. 
Table A7: Coffee UPC Price Summaries

\begin{tabular}{rcccccccc}
\hline \multicolumn{1}{c}{ Brand } & Share & Mean & SD & p10 & p25 & Median & p75 & p90 \\
\hline Peet's & $1.98 \%$ & 10.76 & 1.60 & 8.61 & 9.65 & 10.74 & 11.86 & 12.99 \\
Millstone & $2.17 \%$ & 10.58 & 2.10 & 7.98 & 8.96 & 10.49 & 12.07 & 13.36 \\
Starbucks & $10.92 \%$ & 10.18 & 3.53 & 8.11 & 8.82 & 9.68 & 10.57 & 11.58 \\
Seattle's Best & $1.53 \%$ & 8.89 & 1.95 & 6.76 & 7.60 & 8.78 & 9.92 & 11.04 \\
Dunkin' & $2.70 \%$ & 8.54 & 1.27 & 7.24 & 7.70 & 8.54 & 9.27 & 10.09 \\
New England & $1.40 \%$ & 6.42 & 1.44 & 4.44 & 5.65 & 6.47 & 7.33 & 8.14 \\
Other & $16.05 \%$ & 6.27 & 3.58 & 2.15 & 3.52 & 5.56 & 8.83 & 10.85 \\
Nescafé & $2.32 \%$ & 6.25 & 2.88 & 3.36 & 4.11 & 5.18 & 8.59 & 10.69 \\
Don Francisco's & $1.45 \%$ & 6.00 & 1.88 & 3.90 & 4.58 & 5.38 & 7.26 & 8.84 \\
Folgers & $24.51 \%$ & 5.49 & 4.72 & 2.67 & 3.25 & 4.12 & 5.33 & 7.44 \\
Private Label & $10.64 \%$ & 5.37 & 4.41 & 2.14 & 2.72 & 4.02 & 7.42 & 9.32 \\
Eight O'Clock & $5.27 \%$ & 5.16 & 1.83 & 3.28 & 4.04 & 4.95 & 5.84 & 6.95 \\
Maxwell House & $16.34 \%$ & 4.88 & 3.82 & 2.37 & 2.98 & 3.74 & 4.95 & 9.17 \\
Chock full o'Nuts & $2.70 \%$ & 4.05 & 1.20 & 2.65 & 3.20 & 3.95 & 4.73 & 5.59 \\
\hline
\end{tabular}

Notes: Table presents summary statistics for coffee UPC prices (in January 2001 dollars) by brand. Share is overall revenue share of brand. Each observation represents a store-UPC-week. 
Table A8: Competitive Effects of Walmart Supercenters by Brand: Coffee

\begin{tabular}{|c|c|c|}
\hline & $\begin{array}{c}\text { Log Revenue } \\
\text { (1) }\end{array}$ & $\begin{array}{c}\text { Log Price } \\
(2)\end{array}$ \\
\hline$\# \mathrm{WM} *$ Peet's & $\begin{array}{l}-0.0394 \\
(0.0505)\end{array}$ & $\begin{array}{c}0.00550 \\
(0.00487)\end{array}$ \\
\hline \# WM * Millstone & $\begin{array}{r}-0.00747 \\
(0.0357)\end{array}$ & $\begin{array}{l}-0.00278 \\
(0.00351)\end{array}$ \\
\hline \# WM * Starbucks & $\begin{array}{c}-0.0302 \\
(0.0280)\end{array}$ & $\begin{array}{c}0.00193 \\
(0.00320)\end{array}$ \\
\hline \# WM * Seattle's Best & $\begin{array}{c}0.0202 \\
(0.0343)\end{array}$ & $\begin{array}{c}-0.00107 \\
(0.00315)\end{array}$ \\
\hline \# WM * Dunkin' & $\begin{array}{c}0.0184 \\
(0.0415)\end{array}$ & $\begin{array}{c}0.000534 \\
(0.00433)\end{array}$ \\
\hline \# WM * New England & $\begin{array}{l}-0.0417 \\
(0.0660)\end{array}$ & $\begin{array}{c}0.00681 \\
(0.00758)\end{array}$ \\
\hline \# WM * Nescafé & $\begin{array}{c}-0.204^{* * *} \\
(0.0634)\end{array}$ & $\begin{array}{c}-0.00248 \\
(0.00383)\end{array}$ \\
\hline \# WM * Don Francisco's & $\begin{array}{l}-0.0420 \\
(0.0398)\end{array}$ & $\begin{array}{c}0.00120 \\
(0.00911)\end{array}$ \\
\hline \# WM * Folgers & $\begin{array}{c}-0.0364^{* *} \\
(0.0161)\end{array}$ & $\begin{array}{c}0.00120 \\
(0.00265)\end{array}$ \\
\hline \# WM * Private Label & $\begin{array}{c}-0.0510^{*} \\
(0.0281)\end{array}$ & $\begin{array}{c}-0.00127 \\
(0.00140)\end{array}$ \\
\hline \# WM * Eight O'Clock & $\begin{array}{c}0.0120 \\
(0.0362)\end{array}$ & $\begin{array}{c}0.00109 \\
(0.00439)\end{array}$ \\
\hline \# WM $*$ Maxwell House & $\begin{array}{c}-0.0815^{* * *} \\
(0.0213)\end{array}$ & $\begin{array}{l}-0.00115 \\
(0.00251)\end{array}$ \\
\hline \# WM * Chock full o'Nuts & $\begin{array}{l}-0.0183 \\
(0.0306)\end{array}$ & $\begin{array}{c}0.00276 \\
(0.00622)\end{array}$ \\
\hline \# WM $*$ Other & $\begin{array}{l}-0.0236 \\
(0.0258)\end{array}$ & $\begin{array}{c}0.00200 \\
(0.00189)\end{array}$ \\
\hline
\end{tabular}

Notes: Table presents results of regressing incumbent supermarket log revenues and log prices for coffee on Walmart Supercenter exposure allowing for heterogeneity by brand. WM $=1$ for Supercenter exposures within 5 miles. 6,716,504 store-brand-week observations in column (1) and 85,464,132 store-UPC-week observations in column $(2)^{* * *} \mathrm{p}<0.01,{ }^{* *} \mathrm{p}<0.05,{ }^{*} \mathrm{p}$ $<0.1$. Standard errors clustered by store in parentheses. Markets are IRI designated markets. Standard errors clustered by store in parentheses. Column (1) includes period fixed effects, store-category fixed effects, category trends, market trends, and treatment group trends. Treatment groups are defined by total Supercenter exposures. Markets are IRI designated markets. All trends are quadratic. Column (2) includes store-UPC fixed effects and market-UPC-period fixed effects. 
Table A9: Frozen Dinner UPC Price Summaries

\begin{tabular}{rcccccccc}
\hline \multicolumn{1}{c}{ Brand } & Share & Mean & SD & p10 & p25 & Median & p75 & p90 \\
\hline Amy's Kitchen & $1.50 \%$ & 6.00 & 1.03 & 4.63 & 5.29 & 6.01 & 6.67 & 7.31 \\
Weight Watchers & $9.96 \%$ & 3.80 & 0.94 & 2.65 & 3.06 & 3.70 & 4.43 & 5.03 \\
Stouffer's & $35.93 \%$ & 3.77 & 1.12 & 2.42 & 2.95 & 3.68 & 4.51 & 5.26 \\
Healthy Choice & $9.79 \%$ & 3.68 & 0.87 & 2.62 & 3.04 & 3.58 & 4.25 & 4.88 \\
Other & $14.98 \%$ & 3.60 & 1.47 & 1.77 & 2.42 & 3.51 & 4.56 & 5.52 \\
Kid Cuisine & $2.30 \%$ & 3.51 & 0.69 & 2.67 & 3.01 & 3.46 & 3.94 & 4.45 \\
Boston Market & $2.44 \%$ & 3.49 & 0.94 & 2.40 & 2.84 & 3.34 & 4.05 & 4.78 \\
Marie Callender's & $6.33 \%$ & 3.04 & 0.83 & 2.07 & 2.45 & 2.92 & 3.56 & 4.19 \\
Swanson & $3.59 \%$ & 3.00 & 0.76 & 2.04 & 2.46 & 2.98 & 3.51 & 3.95 \\
Private Label & $2.09 \%$ & 2.99 & 1.35 & 1.73 & 2.30 & 2.85 & 3.30 & 4.27 \\
Michelina's & $4.12 \%$ & 2.23 & 0.69 & 1.55 & 1.69 & 2.03 & 2.64 & 3.19 \\
Banquet & $6.96 \%$ & 1.80 & 0.55 & 1.28 & 1.41 & 1.63 & 2.03 & 2.54 \\
\hline
\end{tabular}

Notes: Table presents summary statistics for frozen dinner UPC prices (in January 2001 dollars) by brand. Share is overall revenue share of brand. Each observation represents a store-UPC-week. 
Table A10: Competitive Effects of Walmart Supercenters by Brand: Frozen Dinner Entrees

\begin{tabular}{|c|c|c|}
\hline & $\begin{array}{c}\text { Log Revenue } \\
\text { (1) }\end{array}$ & $\begin{array}{l}\text { Log Price } \\
\quad(2)\end{array}$ \\
\hline \# WM * Amy's Kitchen & $\begin{array}{c}-0.0806^{* *} \\
(0.0346)\end{array}$ & $\begin{array}{c}-0.00416 \\
(0.00382)\end{array}$ \\
\hline \# WM * Weight Watchers & $\begin{array}{c}0.00965 \\
(0.0272)\end{array}$ & $\begin{array}{c}0.00124 \\
(0.00330)\end{array}$ \\
\hline \# WM * Stouffer's & $\begin{array}{l}-0.0196 \\
(0.0210)\end{array}$ & $\begin{array}{l}-0.00493 \\
(0.00308)\end{array}$ \\
\hline \# WM * Healthy Choice & $\begin{array}{c}-0.0655^{* * *} \\
(0.0238)\end{array}$ & $\begin{array}{l}-0.00144 \\
(0.00294)\end{array}$ \\
\hline$\#$ WM $*$ Kid Cuisine & $\begin{array}{c}-0.108^{* * *} \\
(0.0230)\end{array}$ & $\begin{array}{l}0.000114 \\
(0.00286)\end{array}$ \\
\hline \# WM * Boston Market & $\begin{array}{l}-0.0234 \\
(0.0379)\end{array}$ & $\begin{array}{c}0.00256 \\
(0.00425)\end{array}$ \\
\hline \# WM * Marie Callender's & $\begin{array}{l}-0.0237 \\
(0.0323)\end{array}$ & $\begin{array}{l}-0.00149 \\
(0.00470)\end{array}$ \\
\hline \# WM $*$ Swanson & $\begin{array}{l}-0.0495 \\
(0.0310)\end{array}$ & $\begin{array}{l}-0.00160 \\
(0.00292)\end{array}$ \\
\hline \# WM * Private Label & $\begin{array}{c}-0.141 \\
(0.0946)\end{array}$ & $\begin{array}{l}0.000386 \\
(0.00133)\end{array}$ \\
\hline \# WM * Michelina's & $\begin{array}{c}-0.121^{* * *} \\
(0.0367)\end{array}$ & $\begin{array}{l}-0.00109 \\
(0.00289)\end{array}$ \\
\hline \# WM * Banquet & $\begin{array}{c}-0.0738^{* * *} \\
(0.0266)\end{array}$ & $\begin{array}{l}-0.00227 \\
(0.00402)\end{array}$ \\
\hline \# WM $*$ Other & $\begin{array}{c}0.0157 \\
(0.0269)\end{array}$ & $\begin{array}{r}-0.00413^{* *} \\
(0.00173)\end{array}$ \\
\hline
\end{tabular}

Notes: Table presents results of regressing incumbent supermarket log revenues and log prices for frozen dinner on Walmart Supercenter exposure allowing for heterogeneity by brand. WM $=1$ for Supercenter exposures within 5 miles. 8,068,012 store-brand-week observations in column (1) and 75,992,608 store-UPC-week observations in column $(2)^{* * *} \mathrm{p}<0.01, * * \mathrm{p}<0.05,{ }^{*} \mathrm{p}$ $<0.1$. Standard errors clustered by store in parentheses. Markets are IRI designated markets. Standard errors clustered by store in parentheses. Column (1) includes period fixed effects, store-category fixed effects, category trends, market trends, and treatment group trends. Treatment groups are defined by total Supercenter exposures. Markets are IRI designated markets. All trends are quadratic. Column (2) includes store-UPC fixed effects and market-UPC-period fixed effects. 
Table A11: Competitive Revenue and Price Effects of Walmart Supercenters by Order of Entry

\begin{tabular}{ccccc}
\hline & \multicolumn{2}{c}{ Log Revenue } & \multicolumn{2}{c}{ Log Prices } \\
& $(1)$ & $(2)$ & $(3)$ & $(4)$ \\
\hline \# WM $<5$ Miles & $-0.0603^{* * *}$ & & -0.000744 & \\
& $(0.0131)$ & & $(0.00241)$ & \\
One WM $<5$ Miles & & $-0.0649^{* * *}$ & & -0.000307 \\
Two WMs $<5$ Miles & & $(0.0184)$ & & $(0.00317)$ \\
Three+ WMs $<5$ Miles & & $-0.111^{* * *}$ & 0.000498 \\
& & $(0.0274)$ & & $(0.00533)$ \\
& & $-0.213^{* * *}$ & & -0.0140 \\
& & $(0.0472)$ & & $(0.00861)$ \\
\hline
\end{tabular}

Notes: Table presents results of regressing incumbent supermarket log revenue and log prices on Walmart Supercenter entry by order of exposure. Columns (1) and (2) includes 756,097 storeweek observations and columns (3) and (4) 11,253,196 store-category-week observations. *** p < $0.01,{ }^{* *} \mathrm{p}<0.05,{ }^{*} \mathrm{p}<0.1$. Standard errors clustered by store in parentheses. All specifications control for period fixed effects, store-category fixed effects, category trends, market trends, and treatment group trends. Treatment groups are defined by total Supercenter exposures. Markets are IRI designated markets and category refers to product category. All trends are quadratic. 